\title{
Spotlight on nano-theranostics in South Korea: applications in diagnostics and treatment of diseases
}

This article was published in the following Dove Press journal:

International Journal of Nanomedicine

25 August 2015

Number of times this article has been viewed

\author{
Sangwha Lee ${ }^{1, *}$ \\ Jongsung Kim ${ }^{1, *}$ \\ Chung Wung Bark ${ }^{2}$ \\ Bonghee Lee $^{3}$ \\ Heongkyu Ju ${ }^{4}$ \\ Se Chan Kang ${ }^{5}$ \\ TaeYoung Kim ${ }^{6}$ \\ Moon II Kim ${ }^{6}$ \\ Young Tag $\mathrm{Ko}^{3}$ \\ Jeong-Seok $\mathrm{Nam}^{3}$ \\ Hyon Hee Yoon' \\ Kyu-Sik Yun ${ }^{1,6}$ \\ Young Soo Yoon' \\ Seong Soo A An ${ }^{1,6}$ \\ John Hulme 6
}

'BioNano Sensor Research Center,

${ }^{2}$ Department of Electrical Engineering,

Gachon University, Seongnam-si,

${ }^{3}$ Lee Gil Ya Cancer and Diabetes

Institute, Gachon University, Incheon,

${ }^{4}$ Department of Nano-Physics,

${ }^{5}$ Department of Life Science, Gachon

University, ${ }^{6}$ Department of BioNano

Technology, Gachon BioNano

Research Institute, Seongnam-si,

South Korea

*These authors contributed equally to this work

Correspondence: John Hulme;

Seong Soo A An

Department of BioNano Technology,

Gachon BioNano Research Institute,

Gachon University, 1342 Sungnam-daero,

Sujung-gu, Seongnam-si,

Gyeonggi-do 46I-70I, South Korea

Tel +823 3। 7508550

Fax +82 31 7508755

Email johnhulme2I@gmail.com;

seongaan@gachon.ac.kr
Abstract: From the synergistic integration and the multidisciplinary strengths of the BioNano Sensor Research Center, Gachon Bionano Research Institute, and Lee Gil Ya Cancer and Diabetes Institute, researchers, students, and faculties at Gachon University in collaboration with other institutions in Korea, Australia, France, America, and Japan have come together to produce a special issue on the diverse applications of nano-theranostics in nanomedicine. This special issue will showcase new research conducted by various scientific groups in Gyonggi-do and Songdo/ Incheon, South Korea. The objectives of this special issue are as follows: 1) to bring together and demonstrate some of the latest research results in the field, 2) to introduce new multifunctional nanomaterials and their applications in imaging and detection methods, and 3) to stimulate collaborative interdisciplinary research at both national and international levels in nanomedicine.

Keywords: cancer, imaging and therapeutics, antibacterial, disease, neurodegenerative

\section{Introduction}

Gyeonggi Province in the South Korea under the Gyeonggi Regional Research Center (GRRC) program established the BioNano Sensor Research Center (BSRC) within Gachon Bionano Research Institute (GBRI) at Gachon University to boost research initiatives in bionano sensor technologies in collaboration with Lee Gil Ya Cancer and Diabetes Research Institute. In the last 10 years, bionanotechnology (nanobiotechnology) has emerged as an interdisciplinary subject that utilizes the expertise from various scientific fields to tackle significantly complex biological problems at the micron and nanoscale.

BSRC/GBRI and Lee Gil Ya Cancer and Diabetes Research Institute at Gachon University are strategically located at Songpa-Pangyo-Gwanggyo Valley and Songdo/Incheon, two leading-edge meccas for the future medical industries through creating many synergies from the convergence of bio and medical technologies. The Valley and Songdo would be the pivotal locations, new homes, for the Korean medical industries in the 21st century, including Samsung Biologics (Yeonsu-gu, Incheon, Korea) and Celltrion Inc (Incheon, South Korea). The latest and tailored education and training activities at BSRC/GBRI provides professionals with a modern set of scientific skills suited to the needs of Korean medical industries, thus reducing the time and cost of reeducation of new employees, whilst accelerating the development of new products and the overall competitiveness of medical research conducted in the Songpa-Pang-GwanggyoValley and Songdo-/Incheon areas. Indeed, BSRC/GBRI and Gachon University will contribute to the local economy in Gyeonggi Providence and Incheon for their growth.

BSRC/GBRI's research activities consist of developing and transferring various nanobiotechnologies for detecting and controlling DNA, RNA, proteins, cells, and 
biomaterials in nanoscales, which have been foreseen as driving engines for the future high-technology industries through the convergence of biotechnology (BT), nanotechnology (NT), information technology (IT), and medical technology (MT).

Our aims are to create foundations and stepping stones in performing our faithful role of expanding the technological frontiers through the convergences of various technologies. Interactive collaborations between BSRC/GBRI and the local industries in Gyeonggi Providence would open opportunities for efficient reviews and transfer the latest technologies from BSRC/GBRI to industries. Through collaborations with bioventure companies in biosensors, BT, and NT, the new patents from BSRC will be available to bring forth many commercialized products.

This special issue highlights the properties of newly developed multifunctional nanomaterials and how they can be used to monitor disease states in vivo and in vitro. The purpose of this issue is not only to inform new scientists about advances in nanomedicine, but also to inspire them to seek out avenues of exciting research that may be of future benefit in the treatment and detection of diseases investigated herein.

\section{Research topics}

The current issue covers various material and technological approaches employed by researchers to further our understanding of disease states in nanomedicine. A sample list of the topics covered follows.

\section{Cancer imaging and therapeutics}

Magnetic resonance imaging (MRI) and X-ray computer tomography (CT) are two of the most commonly used imaging techniques in the diagnosis and treatment of hard and soft tumors. Accurate tumor diagnosis using either of these techniques is dependent on the resolution of the machine (strength of the field), the quality of the contrast agents (CAs), and the skill of the operator. To date, the most powerful MRI machines (14 T) in operation can be found in South Korea, ${ }^{1}$ Europe, ${ }^{2}$ and USA. ${ }^{3}$ Like MRI, CT is also a very powerful technique, unfortunately overradiation or stressing of the imaging area is common, resulting in misdiagnosis. ${ }^{4}$ Recently however, researchers in USA and Japan have utilized improvements in NT to realize a CT scanner that can reduce radiation exposure by as much as $95 \%$ in patients. The CT machine can complete a full rotation in $275 \mathrm{~ms}$. Current scanners top out at $325 \mathrm{~ms}$ rotations. In addition to improvements in safety, new types of CAs need to be developed, which are suited to high-speed, high-resolution scanners. To address these new challenges, scientists in Gyeonggi-do, South Korea, have recently been developing new types of silica-based CAs and anticancer delivery vehicles. ${ }^{5}$

\section{Antibacterial agents and neurodegenerative testing}

There are several papers that eloquently show the antibacterial effects of nanoparticles, nanoshells, and nanorods on pathogenic bacteria. ${ }^{6-8}$ However, the gut and skin are principally composed of commensal bacteria outnumbering the pathogenic bacteria by several orders of magnitude. Targeted delivery via complementary antibodies is one way to retain the commensal population, reduce recovery times, and guard against secondary infections. In the age of 1 day genomic mapping, there is no justifiable reason for the nontargeted delivery of cheap and modern powerful antibiotics. Unfortunately, there is no modern economic benefit to do so. Consequently, the number of bacterial-resistant strains found in underdeveloped and developed countries is on the rise. The combinatorial application of nanomaterials and antibiotics against pathogenic bacteria remains a key part in the ongoing economic battle against life-threatening infections. ${ }^{9}$ The application of nanomaterials as delivery vehicles for antibiotics should limit their overmedication in patients, cleaning solutions, bandages, and skin infections. ${ }^{10}$

Neurodegenerative disease is an umbrella term for a range of conditions that primarily affect the neurons in the human brain. Examples of neurodegenerative diseases include Parkinson's, Alzheimer's, Huntington's, and Prion diseases. ${ }^{11}$ A major goal of current clinical research in neurodegenerative diseases is to improve early detection of disease and presymptomatic detection of neuronal dysfunction. Many different approaches are being undertaken to identify biomarkers such as high-resolution imaging, neurophysiological and cognitive testing, biochemical, proteomic, metabolic and gene array profiling of tissue, and biofluids from patients and animals. The ideal biomarker needs to be easy to quantify and measure, reproducible and unaffected by comorbid factors. ${ }^{12}$

\section{Sensors}

Biochemical analyses in a clinical setting are expensive and time-consuming processes. More and more measurements of analytes are performed in various locations, including hospital point-of-care settings, by caregivers in nonhospital settings and by patients themselves. Today, one of the main challenges is the development of methods to perform these rapid in situ analyses. These methods must be sensitive 
and accurate and able to determine various substances with different properties in real-life samples. Modern concept of using electrochemical sensors to determine the concentration of substances and other parameters of biological interest has represented a rapidly expanding field of instrument design since 1962, when Clark and Lyons ${ }^{13}$ invented the first electrochemical biosensor, known as the "enzyme electrode," using the enzyme glucose oxidase to an amperometric electrode for dissolved oxygen. Electrochemical sensors have improved the performance of the conventional analytical tools, eliminated slow preparation and the use of expensive reagents, and provided low-cost analytical tools. As inexpensive, portable, and simple-to-operate analytical tools, electrochemical sensors have had certain advantages over the conventional analytical instruments. In addition, the old-standing problems of electrolyte interference from blood components, namely, albumin and creatinine ( $\mathrm{Cr}$ ), were essentially solved in the clinical and industrial arena back in the 1990s with the introduction of ion and other copolymer selective membranes. ${ }^{14,15}$ Nevertheless, disposable electrochemical sensors continue to offer numerous applications in clinical diagnosis, environmental monitoring, and food analysis. ${ }^{16}$

\section{Papers in this special issue}

Rapid, reliable, and cost-effective monitoring of soft- and hard-tissue replacement postoperatively remains a constant challenge for most clinical establishments. Seo et al reported a new high-speed imaging technique, Synchrotron Radiation
X-ray Computerized Topography (SR-CT), which can monitor the efficacy of various bone-grafting materials for maxillary sinus floor elevation in human beings. Although the SR $\mu$-CT technique could not distinguish complex-shaped tissues, the bone regeneration pattern and spatial organization of hard tissues were visualized. Yi et al presented a gadolinium (Gd)-based dual imaging colloidal hybrid silica nanoparticle. Fluorescence and MRI data showed that the Gd-silica nanohybrids can be circulated in vivo with a broad distribution in mice. Interestingly, the multifunctional nanoparticles showed enhanced magnetic resonance relaxivity compared to previously reported materials. Kim et al and Dubey et al investigated the anticancer properties of self-assembled molecular bowls containing phenanthrene-based donor ${ }^{5}$ and RU(II) acceptors and ellagic acid encapsulated nanosize metalla. Molecular bowel 6 was selectively cytotoxic to gastric cancer cells in the first paper, whereas ellagic acid metalla (compound 10) exhibited an inhibitory effect for cancer via granulocyte-colony stimulating factor (G-CSF) induction and Rantes inhibition in the second one. It was found that priming with ellagic acid and compound $\mathbf{1 0}$ enhanced the tumoricidal activity, by $55.1 \%$ (with $2 \mu \mathrm{M}$ of ellagic acid) and $58.6 \%$ (with $2 \mu \mathrm{M}$ of compound $\mathbf{1 0}$ ) as compared to control macrophages. In order to confirm these results, researchers investigated the mRNA expression of G-CSF and Rantes in the macrophage RAW264.7 cell line by RT-PCR. G-CSF was increased by compound $\mathbf{1 0}(\sim 2.1$ times vs control) and Rantes was decreased ( $\sim 0.4$ times vs control) with respect
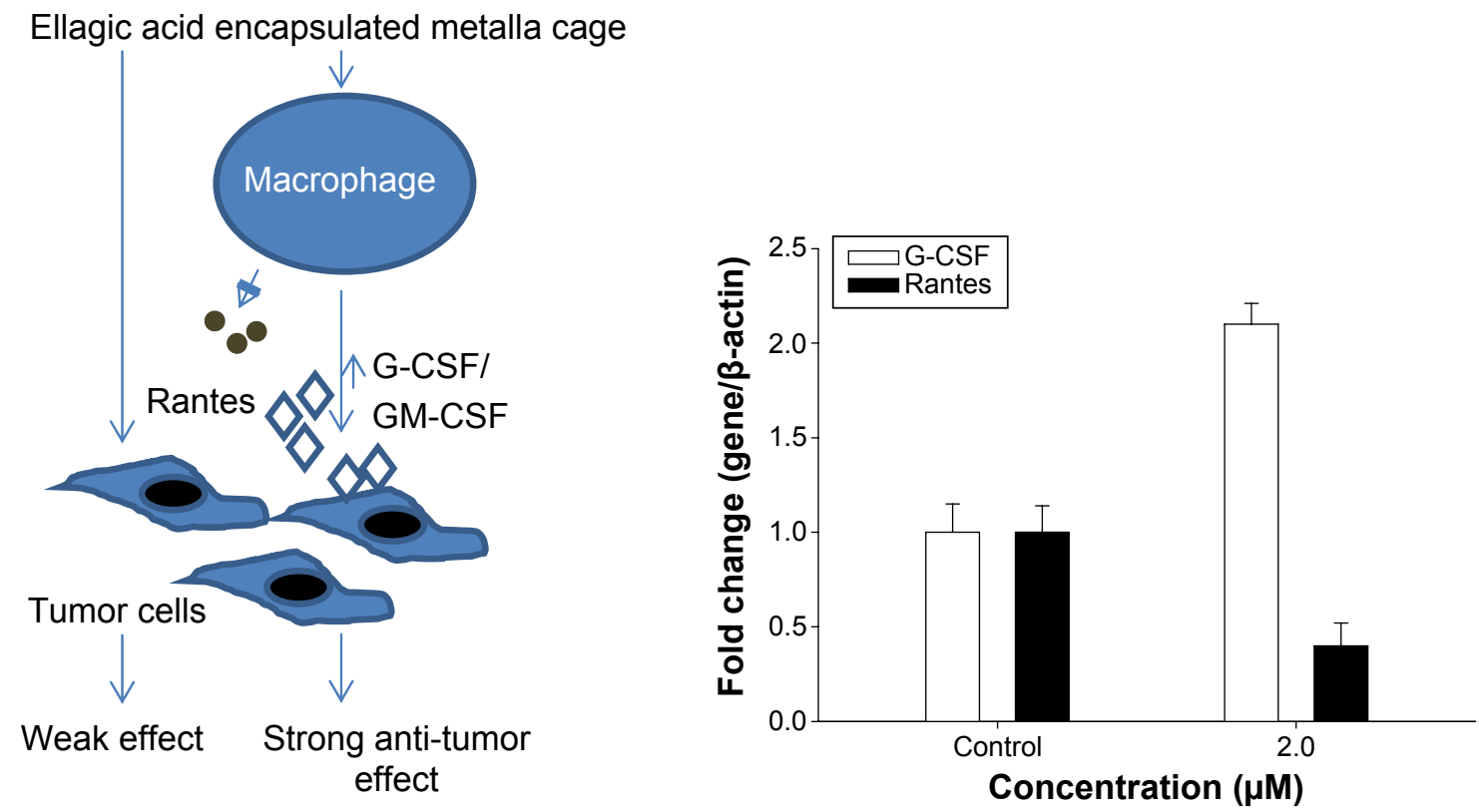

Figure I Schematic representing the proposed events and G-CSF and Rantes gene expression in RAW264.7 macrophages with ellagic acid (control) and compound. Abbreviations: G-CSF, granulocyte-colony stimulating factor; GM-CSF, Granulocyte-macrophage colony-stimulating factor. 
to the mRNA levels. The results in Figure 1 confirm ellagic acid encapsulated into a nanosized metalla cage inhibited tumor cells through modulation of G-CSF and Rantes in macrophages at the level of both protein and mRNA.

Joo et al examined the antitumor efficacy of fibrinogen (Fbg) $\mu$-spheres loaded with doxorubicin (DOX). In this study, researchers used a smart mechanism to trigger the localized release of DOX in an acidic environment. Microspheres with longer linker lengths bound rapidly to cancer cells. In a study by Kang et al the tumor suppression abilities of anionic and cationic lipid-coated Au nanorods and spheres were investigated. An interesting study on the comparative hyperthermia effects of antibody (Erbitux, [ERB]) conjugated silica-gold nanoshells (SGNS) on epithelial tumor cells was conducted by Lee et al. ERB-coated SGNS showed excellent therapeutic efficacy under near infra-red irradiation.

Tung et al evaluated the volatile organic sensing capabilities of a chemiresistive nanocomposite material composed of magnetic nanoparticles $\left(\mathrm{Fe}_{3} \mathrm{O}_{4}\right)$ and conducting polymers (Poly[3,4-ethylenedioxythiophene] [PEDOT]) and its potential role as a breathalyzer for the detection of lung cancer. $\mathrm{Wu}$ et al provided a comprehensive and updated review of the applications of graphene oxide (GO) in cancer imaging and therapeutics; Jang et al presented a brief but intriguing review on cancer stem cells (CSCs) as potential therapeutic targets. The authors discuss the recent advances in the targeting of notch signaling pathways in CSCs and CSC markers, CD44 and CD133.

The paper by Zhong et al investigates the antibacterial properties of $\mathrm{GO}$ modified $\mathrm{ZnO}$ nanoparticles were investigated. The researchers present a novel antibacterial mechanism for the formation of reactive oxygen species using the substrate. Govindaraju et al provided a detailed investigation regarding the antibacterial properties of broadband UV and laser irradiated functionalized gold nanoparticles on Escherichia coli. Ramasamy et al examined the effects of modifying the surface of gold nanorods with silica on the growth and viability of pathogenic E. coli O157:H7. $\mathrm{Bu}$ et al presented a paper in which the characteristics of $\mathrm{Ag}_{\text {core }} \mathrm{Au}_{\text {shell }}$ nanoparticles as SERS substrates for the sensitive detection of dopamine were investigated. The authors propose that dopamine can act as a molecular bridge between nanoparticles, which can induce a profound influence on the SERS activity of colloidal systems. Figure 2 illustrates the $\mathrm{pH}$-dependent interaction of the $\mathrm{Ag}_{\text {core }} \mathrm{Au}_{\text {shell }}$ NPs with DA molecules at various $\mathrm{pH}$ ranges. Kim et al utilized dopaminefunctionalized $\mathrm{InP} / \mathrm{ZnS}$ quantum dots (QDs) as fluorescence probes for the detection of adenosine in microfluidic chips. Adenosine is an inhibitory neurotransmitter and a signaling molecule that is involved in energy production in the form of ATP at the molecular level. In this investigation, researchers use adenosine to competitively inhibit the binding of zinc ions to different types of dopamine conjugated QDs. It was found that the proposed fluorescence bio-probe has potential application in biological systems for the detection of adenosine in solution and serum at low concentration levels. In another paper by Kim et al the fluorescence quenching of cysteine-capped InP/ZnS QDs by dopamine and ascorbic acid was investigated. The quenching of the QDs was significantly higher for dopamine than ascorbic

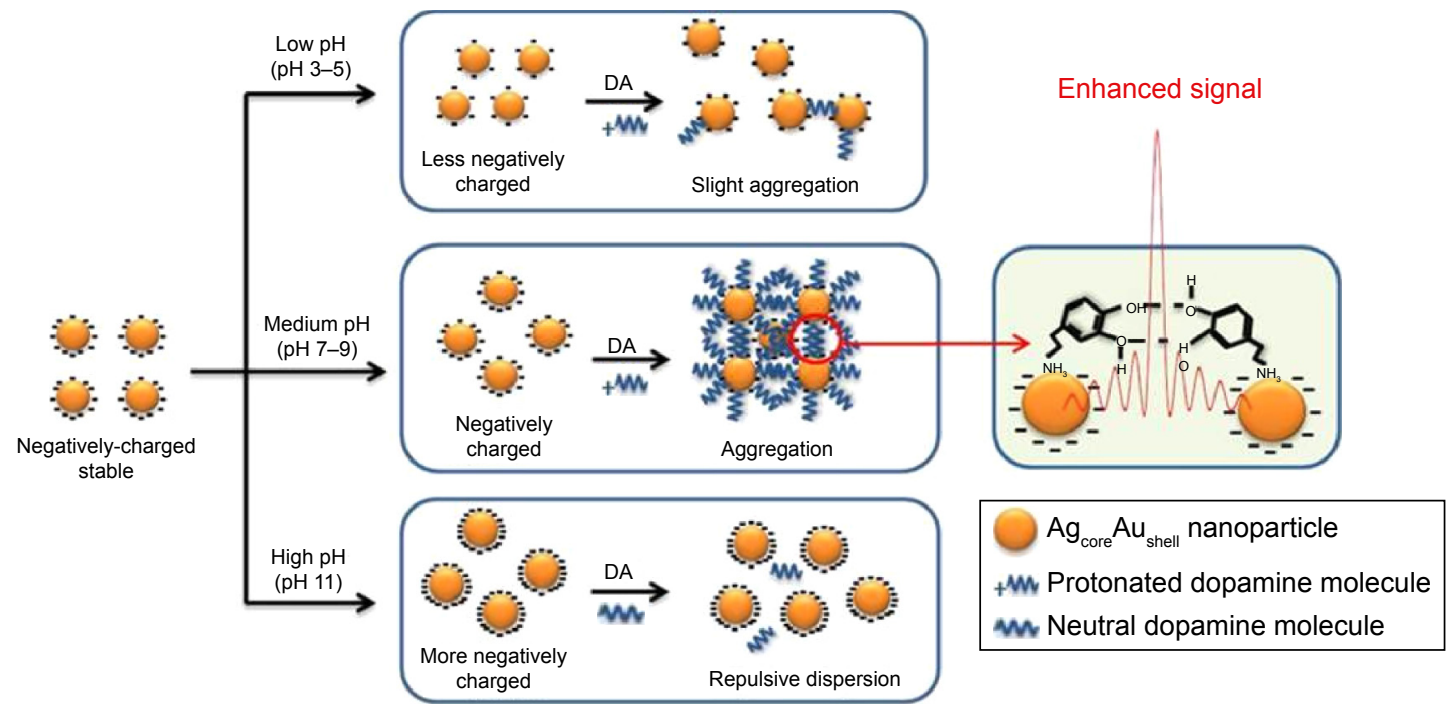

Figure 2 The aggregation behavior of $\mathrm{Ag}_{\text {core }} \mathrm{Au}_{\text {shell }} \mathrm{NPs}$ with and without DA molecules at various $\mathrm{pH}$ ranges. Abbreviations: NPs, nanoparticles; DA, dopamine. 
acid. The analytical performance of this assay for DA was assessed, and it was found that the limit of detection was $875 \mathrm{pM}$. The following paper by Subbiah et al examined the effects of different concentrations of silver nanoparticles on the physicomechanical responses of human alveolar epithelial cells (A549), mouse fibroblasts (NIH3T3), and human bone marrow stromal cells (hBMSC; HS-5). The authors reported that alterations in cellular morphology were directly proportional to the level of AgNP-induced cytotoxicity. AgNPs, treatment increased the surface roughness $(P<0.001)$ but more importantly the stiffness $(P<0.001)$ of all the cell lines investigated.

Lim et al combined a Multimer Detection System with magnetic microparticles to enhance the detection of aggregated prion proteins in blood taken from preclinical and clinical sheep in a double-blind study. Figure 3 illustrates how a multimer assay works.

This issue also contains several reports that employ different types of electrochemical and optical sensing strategies in the detection of clinical metabolites, glucose, serum albumin, Cr, urea and sexually transmitted diseases (STDs). The paper by Das et al examines the stability and glucose sensitivity of three different spherulitic copper-copper oxide nanostructures deposited on multi-walled carbon nanotube and modified on indium tin oxide electrodes. In the paper, "Optical birefringence of liquid crystals for label-free optical biosensing diagnosis" by Nguyen et al the authors elegantly employed liquid crystals and a gold interface in the label free detection of serum albumin. The approach has the potential to generate accurate kinetic data without interference from fluorophores or additional labels. In the paper by Nguyen et al an optical fiber sensor based on surface plasmon excitation was used to detect Fbg extracted from human blood plasma. The authors demonstrated a real-time quantitative detection of Fbg concentrations with a limit of detection of $10 \mathrm{ng} / \mathrm{mL}$. In addition, the sensor surface was easily regenerated by washing the fiber head in acidified imidazole (IM) solution for 2 minutes.

Nanda et al demonstrated a new method for the detection of $\mathrm{Cr}$ by combining the peroxidase sensing capabilities of dichlorofluorescein diacetate with a functional porous polymer. Das et al contributed a paper in which a highly sensitive electrochemical biosensor composed of sulfonated graphene/ polyaniline film was developed for urea analysis. In the paper "Ultrafast sonochemical synthesis of protein-inorganic nanoflowers" by Batule et al nanoflowers containing the model protein laccase and copper sulfate were synthesized in $<5$ minutes. The resulting laccase nanoflowers were used in the detection of epinephrine.

Jee et al reported on the development of a new and rapid manufacturing process. The glass platform produced by the bubbling process exhibited uniform and optimal surface chemistry. The process was highly flexible and well suited to modern DNA microarray-based diagnostics and the detection

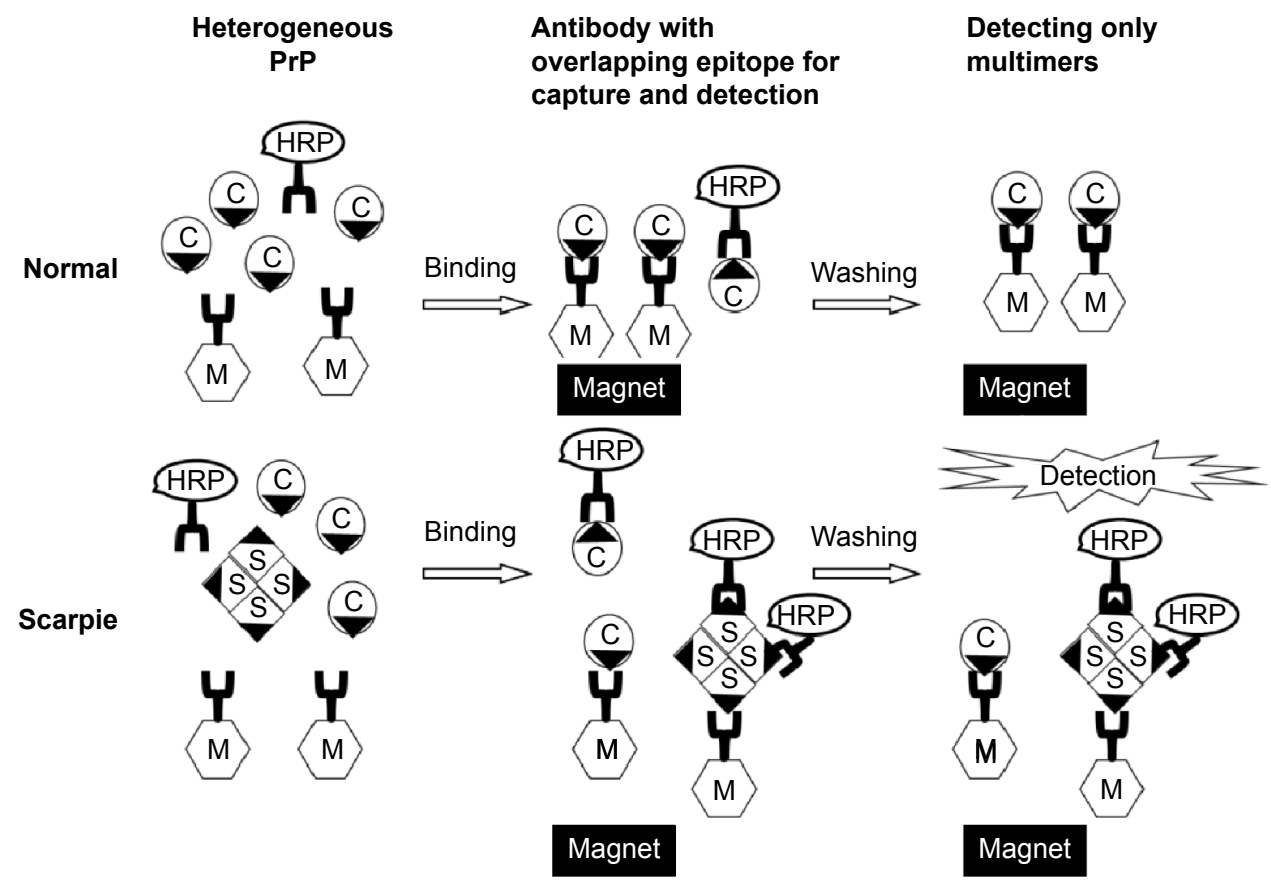

Figure 3 Schematic diagram of the multimer detection system (MDS). 
of STDs. It was found that glass surfaces modified via the bubbling method prior spotting exhibited higher intensities and smaller variations in signal intensity than glass surfaces modified using traditional dip-coating methods. An STD microarray fabricated using the bubbling method produced the highest signal intensity (8.812) and minimum standard deviation (0.0692) due to the optimized (3-Aminopropyl) triethoxysilane surface.

\section{Conclusion}

Twenty-five papers bring together some of the latest collaborative research in nanomedicine from a variety of clinical, academic, and industrial international institutions and companies. We hope that this special issue will encourage further research in this new field.

\section{Acknowledgments}

This work was supported by the GRRC program of Gyeonggi Province (GRRC Gachon 2015-B04, Development of Microfluidic Chip for Diagnosing Diseases). The authors gratefully acknowledge the other contributions of the following scientists: Assistant Professor Yong-Gun Kim, Kyungpook National University, Korea; Professor Ki-Whan Chi, University of Ulsan, Ulsan Korea; Professor DK Yi, Myongji University, Korea; Ms Siying Wu and So-Hee Kim are thanked for proof reading and valuable comments in the paper.

\section{Disclosure}

The authors report no conflicts of interest in this work.

\section{References}

1. Park J, Wicki J, Knoblaugh SE, Chamberlain JS, Lee D. Multi-parametric MRI at $14 \mathrm{~T}$ for muscular dystrophy mice treated with AAV vectormediated gene therapy. PLoS One. 2015;10(4): 0124914.

2. Federau C, Hagmann P, Maeder P, et al. Dependence of brain intravoxel incoherent motion perfusion parameters on the cardiac cycle. PLoS One. 2013;8(9):e72856.
3. Zhu X, Qiao H, Du F, et al. Quantitative imaging of energy expenditure in human brain. Neuroimage. 2012;60(4):2107-2117.

4. Goldstein SA, Evangelista A, Abbara S, et al. Multimodality imaging of diseases of the thoracic aorta in adults: from the American Society of Echocardiography and the European Association of Cardiovascular Imaging. J Am Soc Echocardiogr. 2015;28(2):119-182.

5. Dubey A, Min JW, Koo HJ, et al. Anticancer potency and multidrugresistant studies of self-assembled arene-ruthenium metallarectangles. Chemistry. 2013;19(35):11622-11628.

6. Davies SC, Fowler T, Watson J, Livermore DM, Walker D. Annual Report of the Chief Medical Officer: infection and the rise of antimicrobial resistance. Lancet. 2013;381(9878):1606-1609.

7. Howard SJ, Catchpole M, Watson J, Davies SC. Antibiotic resistance: global response needed. Lancet Infect Dis. 2013;13(12):1001-1003.

8. Huh AJ, Kwon YJ. "Nanoantibiotics": a new paradigm for treating infectious diseases using nanomaterials in the antibiotics resistant era. J Control Release. 2011;156(2):128-145.

9. Mehmood S, Rehman MA, Ismail HA, Mirza B, Bhatti AS. Significance of post growth processing of $\mathrm{ZnO}$ nanostructures on antibacterial activity against gram-positive and gram-negative bacteria. Int $J$ Nanomedicine. 2015;10:4521-4533.

10. Li LH, Yen MY, Ho CC, et al. Non-cytotoxic nanomaterials enhance antimicrobial activities of cefmetazole against multidrug-resistant Neisseria gonorrhoeae. PLoS One. 2013;8(5):e64794.

11. Aguzzi A, Nuvolone M, Zhu C. The immunobiology of prion diseases. Nat Rev Immunol. 2013;13:888-902.

12. Sy M, Kitazawa M, Medeiros R, et al. Inflammation induced by infection potentiates tau pathological features in transgenic mice. Am J Pathol. 2011;178(6):2811-2822.

13. Windmiller JR, Wang J. Wearable electrochemical sensors and biosensors: a review. Electroanalysis. 2013;25(1):29-46.

14. Bandodkar AJ, Wang J. Non-invasive wearable electrochemical sensors: a review. Trends Biotechnol. 2014;32(7):363-371.

15. Grieshaber D, MacKenzie R, Voros J, Reimhult E. Electrochemical biosensors - sensor principles and architectures. Sensors. 2008; 8:1400-1458.

16. Shao Y, Wang J, Wu H, Liu J, Aksay IA, Lin Y. Graphene based electrochemical sensors and biosensors: a review. Electroanalysis. 2010;22(10): 1027-1036.
International Journal of Nanomedicine

\section{Publish your work in this journal}

The International Journal of Nanomedicine is an international, peerreviewed journal focusing on the application of nanotechnology in diagnostics, therapeutics, and drug delivery systems throughout the biomedical field. This journal is indexed on PubMed Central, MedLine, CAS, SciSearch ${ }^{\circledR}$, Current Contents ${ }^{\circledR} /$ Clinical Medicine,

\section{Dovepress}

Journal Citation Reports/Science Edition, EMBase, Scopus and the Elsevier Bibliographic databases. The manuscript management system is completely online and includes a very quick and fair peer-review system, which is all easy to use. Visit http://www.dovepress.com/ testimonials.php to read real quotes from published authors. 